\title{
Gravitational cubic interactions for a massive mixed-symmetry gauge field
}

\author{
Yu. M. Zinoviev* \\ Institute for High Energy Physics \\ Protvino, Moscow Region, 142280, Russia
}

\begin{abstract}
In a recent paper [1] cubic gravitational interactions for a massless mixed symmetry field in $A d S$ space have been constructed. In the current paper we extend these results to the case of massive field. We work in a Fradkin-Vasiliev approach and use frame-like gauge invariant description for massive field which works in $(A) d S$ spaces with arbitrary values of cosmological constant including flat Minkowski space. In this, massless limit in $A d S$ space coincides with the results of [1] while we show that it is impossible to switch on gravitational interaction for massless field in $d S$ space.
\end{abstract}

\footnotetext{
*E-mail address: Yurii.Zinoviev@ihep.ru
} 


\section{Introduction}

In a recent paper [1] cubic gravitational interactions for simplest mixed symmetry field (hook) have been investigated using a number of different approaches, namely:

- direct construction off all possible cubic vertices using modified 1 and $1 / 2$ order formalism (similar construction for spin 3 cubic vertices see [2]);

- Fradkin-Vasiliev approach [3, 4] applied to Alkalaev-Shaynkman-Vasiliev (ASV) description of massless hook in $A d S$ space [5, 6, 7];

- Fradkin-Vasiliev approach applied to Stueckelberg description of hook [8, 9] that differs from the ASV one by the presence of some Stueckelberg fields;

- cohomological approach [10, 11] applied to Stueckelberg description.

While the results of different approaches completely agree, it turns out that the most simple and straightforward way to construct interactions is to use Fradkin-Vasiliev approach that initially was formulated for investigation of gravitational interactions for massless partialces in $A d S$ space [3, 4] and then was successfully applied to more general interactions (see e.g. [12, 13, 14]). Let us briefly recall main steps of the procedure.

- We begin with frame-like gauge invariant formulation with the known set of fields and gauge transformations. For each field (both physical and auxiliary) we construct gauge invariant object that we will generically call curvature.

- Then we rewrite free Lagrangian as an expression quadratic in these gauge invariant curvatures. In general such expression will contain higher derivatives terms so we have to adjust coefficients so that all such terms cancel.

- Now we add quadratic corrections to free curvatures supplemented with appropriate corrections to gauge transformations so that variations of deformed curvatures were proportional to the free ones.

- At last we replace free curvatures in the Lagrangian with the deformed ones and adjust coefficients so that all variations vanish on shell. This in turn means that off shell all variations can be compensated by additional corrections to gauge transformations.

As it is clear from this description two main ingredients of such approach are gauge invariance and frame-like formalism [15, 16, 17]. But during a few last years we have seen that there exists frame-like gauge invariant description for massive fields both symmetric [18, 19] as well as for mixed symmetry ones [8, 9, 20, 21]. Moreover such description nicely works both in flat Minkowski space as well as in $(A) d S$ space with arbitrary value of cosmological constant, including all possible massless and partially massless limits. Thus it seems natural to use Fradkin-Vasiliev approach applied to frame-like gauge invariant description for investigations of possible interactions for massive and/or massless particles. In our recent paper [22] we have shown how such procedure works in the case of electromagnetic interactions of massive hook, while the aim of the current paper is to extend the results of [1] to the case of massive hook. 
In the next section we will give all necessary information on the free hook including free Lagrangian, gauge transformations and gauge invariant curvatures. Moreover we will show that using partial gauge fixing one can obtain a simple description for massive hook directly related with ASV description for massless one. One of the lessons from [1] is that at least for the particular hook case such partial gauge fixing "commute" with the switching on an interaction so we may freely use it to simplify calculations without any lost of generality. Then in Section 2 we consider application of the general procedure to the gravitational interactions for massive hook including massless limit in $A d S$ space, while the investigation of massless case in $d S$ space which turns out to be special moved into Appendix.

Notations and conventions. We work in $(A) d S$ space with $d \geq 4$ dimensions. We will use notation $e_{\mu}{ }^{a}$ for background (non-dynamical) frame of $(A) d S$ space and $D_{\mu}$ for $(A) d S$ covariant derivatives normalized so that

$$
\left[D_{\mu}, D_{\nu}\right] \xi^{a}=-\kappa e_{[\mu}{ }^{a} \xi_{\nu]}, \quad \kappa=\frac{2 \lambda}{(d-1)(d-2)}
$$

We use Greek letters for world indices and Latin letters for local ones. Surely, using frame $e_{\mu}{ }^{a}$ and its inverse $e^{\mu}{ }_{a}$ one can freely convert world indices into local ones and vice-verse and we indeed will use such conversion whenever convenient. But separation of world and local indices plays very important role in a frame-like formalism. In particular, all terms in the Lagrangians can be written as a product of forms, i.e. as expressions completely antisymmetric on world indices and this property greatly simplifies all calculations. For that purpose we will often use notations $\left\{\begin{array}{l}\mu \nu \\ a b\end{array}\right\}=e^{\mu}{ }_{a} e_{b}^{\nu}-e^{\nu}{ }_{a} e^{\mu}{ }_{b}$ and so on.

\section{Kinematics}

Frame-like gauge invariant description [8, 9] requires four pairs of physical and auxiliary fields: $\left(\Omega_{\mu}{ }^{a b c}, \Phi_{\mu \nu}{ }^{a}\right),\left(\Omega_{\mu}{ }^{a b}, f_{\mu}{ }^{a}\right),\left(C^{a b c}, C_{\mu \nu}\right)$ and $\left(B^{a b}, B_{\mu}\right)$. Free Lagrangian describing massive particle in $(A) d S$ space has the form:

$$
\begin{aligned}
& \mathcal{L}_{0}=-\frac{3}{4}\left\{{ }_{a b}^{\mu \nu}\right\} \Omega_{\mu}{ }^{a c d} \Omega_{\nu}{ }^{b c d}+\frac{1}{4}\left\{\begin{array}{l}
\mu \nu \alpha \beta \\
a b c d
\end{array}\right\} \Omega_{\mu}{ }^{a b c} D_{\nu} \Phi_{\alpha \beta}{ }^{d}+ \\
& +\frac{1}{2}\left\{{ }_{a b}^{\mu \nu}\right\} \Omega_{\mu}{ }^{a c} \Omega_{\nu}{ }^{b c}-\frac{1}{2}\left\{\begin{array}{c}
\mu \nu \alpha \\
a b c
\end{array}\right\} \Omega_{\mu}{ }^{a b} D_{\nu} f_{\alpha}{ }^{c}- \\
& -\frac{1}{6} C_{a b c}{ }^{2}+\frac{1}{6}\left\{{ }_{a b c}^{\mu \nu \alpha}\right\} C^{a b c} D_{\mu} C_{\nu \alpha}+\frac{1}{4} B_{a b}{ }^{2}-\frac{1}{2}\left\{\begin{array}{c}
\mu \nu \\
a b
\end{array}\right\} B^{a b} D_{\mu} B_{\nu}+ \\
& +m_{1}\left[\left\{\begin{array}{c}
\mu \nu \\
a b
\end{array}\right\} \Omega_{\mu}{ }^{a b c} f_{\nu}{ }^{c}+\left\{\begin{array}{c}
\mu \nu \alpha \\
a b c
\end{array}\right\} \Omega_{\mu}{ }^{a b} \Phi_{\nu \alpha}{ }^{c}\right]+ \\
& +m_{2}\left[\left\{\begin{array}{c}
\mu \nu \alpha \\
a b c
\end{array}\right\} \Omega_{\mu}{ }^{a b c} C_{\nu \alpha}+\left\{\begin{array}{l}
\mu \nu \\
a b
\end{array}\right\} C^{a b c} \Phi_{\mu \nu}^{c}\right]+ \\
& +2 \tilde{m}_{2}\left[\left\{\begin{array}{c}
\mu \nu \\
a b
\end{array}\right\} \Omega_{\mu}{ }^{a b} B_{\nu}+e^{\mu}{ }_{a} B^{a b} f_{\mu}{ }^{b}\right]+\tilde{m}_{1}\left\{\begin{array}{c}
\mu \nu \\
a b
\end{array}\right\} B^{a b} C_{\mu \nu}
\end{aligned}
$$

Here

$$
8 m_{1}{ }^{2}-24 m_{2}^{2}=-3(d-3) \kappa, \quad \tilde{m}_{1,2}=\sqrt{\frac{(d-2)}{(d-3)}} m_{1,2}
$$

The Lagrangian is invariant under the following set of gauge transformations:

$$
\delta_{0} \Phi_{\mu \nu}{ }^{a}=D_{[\mu} z_{\nu]}{ }^{a}+\eta_{\mu \nu}{ }^{a}+\frac{2 m_{1}}{3(d-3)} e_{[\mu}{ }^{a} \xi_{\nu]}+\frac{4 m_{2}}{(d-3)} e_{[\mu}{ }^{a} \zeta_{\nu]}
$$




$$
\begin{aligned}
\delta_{0} \Omega_{\mu}{ }^{a b c} & =D_{\mu} \eta^{a b c}+\frac{4 m_{1}}{3(d-3)} e_{\mu}{ }^{[a} \eta^{b c]} \\
\delta_{0} f_{\mu}{ }^{a} & =D_{\mu} \xi^{a}+\eta_{\mu}{ }^{a}+4 m_{1} z_{\mu}{ }^{a}+\frac{4 \tilde{m}_{2}}{(d-2)} e_{\mu}{ }^{a} \Lambda \\
\delta_{0} \Omega_{\mu}{ }^{a b} & =D_{\mu} \eta^{a b}-2 m_{1} \eta_{\mu}{ }^{a b} \\
\delta_{0} C_{\mu \nu} & =D_{[\mu} \zeta_{\nu]}-2 m_{2} z_{[\mu \nu]}, \quad \delta_{1} C^{a b c}=6 m_{2} \eta^{a b c} \\
\delta_{0} B_{\mu} & =D_{\mu} \Lambda+2 \tilde{m}_{2} \xi_{\mu}+4 \tilde{m}_{1} \zeta_{\mu}, \quad \delta_{0} B^{a b}=-4 \tilde{m}_{2} \eta^{a b}
\end{aligned}
$$

As the relation on the parameters $m_{1,2}$ clearly shows for non-zero values of cosmological constant $\kappa$ it is not possible to set both $m_{1}$ and $m_{2}$ equal to zero simultaneously. In $A d S$ space $(\kappa<0)$ one can set $m_{2}=0$. In this, the whole system decomposes into two disconnected subsystems. One of them with the Lagrangian and gauge transformations:

$$
\begin{aligned}
& \mathcal{L}_{0}=-\frac{3}{4}\left\{\begin{array}{c}
\mu \nu \\
a b
\end{array}\right\} \Omega_{\mu}{ }^{a c d} \Omega_{\nu}{ }^{b c d}+\frac{1}{4}\left\{\begin{array}{c}
\mu \nu \alpha \beta \\
a b c d
\end{array}\right\} \Omega_{\mu}{ }^{a b c} D_{\nu} \Phi_{\alpha \beta}{ }^{d}+ \\
&+\frac{1}{2}\left\{\begin{array}{c}
\mu \nu \\
a b
\end{array}\right\} \Omega_{\mu}{ }^{a c} \Omega_{\nu}{ }^{b c}-\frac{1}{2}\left\{\begin{array}{l}
\mu \nu \alpha \\
a b c
\end{array}\right\} \Omega_{\mu}{ }^{a b} D_{\nu} f_{\alpha}{ }^{c}- \\
&+m_{1}\left[\left\{\begin{array}{c}
\mu \nu \\
a b
\end{array}\right\} \Omega_{\mu}{ }^{a b c} f_{\nu}{ }^{c}+\left\{\begin{array}{c}
\mu \nu \alpha \\
a b c
\end{array}\right\} \Omega_{\mu}{ }^{a b} \Phi_{\nu \alpha}{ }^{c}\right] \\
& \delta_{0} \Phi_{\mu \nu}{ }^{a}= D_{[\mu} z_{\nu]}{ }^{a}+\eta_{\mu \nu}{ }^{a}+\frac{2 m_{1}}{3(d-3)} e_{[\mu}{ }^{a} \xi_{\nu]} \\
& \delta_{0} \Omega_{\mu}{ }^{a b c}=D_{\mu} \eta^{a b c}+\frac{4 m_{1}}{3(d-3)} e_{\mu}{ }^{[a} \eta^{b c]} \\
& \delta_{0} f_{\mu}^{a}=D_{\mu} \xi^{a}+\eta_{\mu}{ }^{a}+4 m_{1} z_{\mu}{ }^{a} \\
& \delta_{0} \Omega_{\mu}^{a b}=D_{\mu} \eta^{a b}-2 m_{1} \eta_{\mu}{ }^{a b}
\end{aligned}
$$

corresponds to massless representation of $A d S$ group (which differs from that of Poincare group [23, 24, 25]), while the other one just gives gauge invariant description of massive antisymmetric second rank tensor. In turn, in $d S$ space one can set $m_{1}=0$. In this case the whole system also decomposes into two disconnected subsystems. One of them with the Lagrangian and gauge transformations:

$$
\begin{aligned}
\mathcal{L}_{0}= & -\frac{3}{4}\left\{\begin{array}{c}
\mu \nu \\
a b
\end{array}\right\} \Omega_{\mu}{ }^{a c d} \Omega_{\nu}{ }^{b c d}+\frac{1}{4}\left\{\begin{array}{c}
\mu \nu \alpha \beta \\
a b c d
\end{array}\right\} \Omega_{\mu}{ }^{a b c} D_{\nu} \Phi_{\alpha \beta}{ }^{d}+ \\
& -\frac{1}{6} C_{a b c}{ }^{2}+\frac{1}{6}\left\{\begin{array}{c}
\mu \nu \alpha \\
a b c
\end{array}\right\} C^{a b c} D_{\mu} C_{\nu \alpha}+ \\
& +m_{2}\left[\left\{\begin{array}{c}
\mu \nu \alpha \\
a b c
\end{array}\right\} \Omega_{\mu}^{a b c} C_{\nu \alpha}+\left\{\begin{array}{c}
\mu \nu \\
a b
\end{array}\right\} C^{a b c} \Phi_{\mu \nu}{ }^{c}\right] \\
\delta_{0} \Phi_{\mu \nu}{ }^{a}= & D_{[\mu} z_{\nu]}^{a}+\eta_{\mu \nu}{ }^{a}+\frac{4 m_{2}}{(d-3)} e_{[\mu}{ }^{a} \zeta_{\nu]}, \quad \delta_{0} \Omega_{\mu}{ }^{a b c}=D_{\mu} \eta^{a b c} \\
\delta_{0} C_{\mu \nu}= & D_{[\mu} \zeta_{\nu]}-2 m_{2} z_{[\mu \nu]}, \quad \delta_{1} C^{a b c}=6 m_{2} \eta^{a b c}
\end{aligned}
$$

corresponds to massless representation of $d S$ group, while the other one describes a so called partially massless spin 2 particle. 
In our recent paper [22] we have investigated electromagnetic interactions for the same massive mixed symmetry field. We have shown that it is impossible to take a limit $m_{1} \rightarrow 0$ without switching off minimal $\mathrm{e} / \mathrm{m}$ interactions, while nothing prevents one from taking a limit $m_{2} \rightarrow 0$. It turns out that the situation with gravitational interactions is the same. Namely, in a very recent paper [1] cubic gravitational interactions for the case $m_{2}=0$ where constructed, while in Appendix A of the current paper we consider the case $m_{1}=0$ and show that it is impossible to switch on gravitational interaction. Thus in the rest of the paper we will always assume that $m_{1} \neq 0$.

Let us return to the general massive case. Having in our disposal explicit form of the gauge transformations we can construct gauge invariant objects (curvatures) for all eight fields (both physical and auxiliary):

$$
\begin{aligned}
\mathcal{R}_{\mu \nu}{ }^{a b c} & =D_{[\mu} \Omega_{\nu]}^{a b c}+\frac{4 m_{1}}{3(d-3)} e_{[\mu}^{[a} \Omega_{\nu]}{ }^{b c]}+\frac{4 m_{2}}{3(d-3)} e_{[\mu}^{[a} C_{\nu]}{ }^{b c]} \\
\mathcal{T}_{\mu \nu \alpha}{ }^{a} & =D_{[\mu} \Phi_{\nu \alpha]}{ }^{a}-\Omega_{[\mu, \nu \alpha]}{ }^{a}+\frac{2 m_{1}}{3(d-3)} e_{[\mu}{ }^{a} f_{\nu, \alpha]}+\frac{4 m_{2}}{(d-3)} e_{[\mu}{ }^{a} C_{\nu \alpha]} \\
\mathcal{F}_{\mu \nu}{ }^{a b} & =D_{[\mu} \Omega_{\nu]}^{a b}+2 m_{1} \Omega_{[\mu, \nu]}{ }^{a b}-\frac{2 \tilde{m}_{2}}{(d-2)} e_{[\mu}^{[a} B_{\nu]}{ }^{b]} \\
T_{\mu \nu}{ }^{a} & =D_{[\mu} f_{\nu]}^{a}-\Omega_{[\mu, \nu]}{ }^{a}-4 m_{1} \Phi_{\mu \nu}{ }^{a}+\frac{4 \tilde{m}_{2}}{(d-2)} e_{[\mu}{ }^{a} B_{\nu]} \\
\mathcal{C}_{\mu}{ }^{a b c} & =D_{\mu} C^{a b c}-6 m_{2} \Omega_{\mu}{ }^{a b c}-\frac{2 \tilde{m}_{1}}{(d-2)} e_{\mu}{ }^{[a} B^{b c]} \\
\mathcal{C}_{\mu \nu \alpha} & =D_{[\mu} C_{\nu \alpha]}-C_{\mu \nu \alpha}+2 m_{2} \Phi_{[\mu \nu, \alpha]} \\
\mathcal{B}_{\mu}{ }^{a b} & =D_{\mu} B^{a b}+4 \tilde{m}_{2} \Omega_{\mu}{ }^{a b}+\frac{4 \tilde{m}_{1}}{3} C_{\mu}{ }^{a b} \\
\mathcal{B}_{\mu \nu} & =D_{[\mu} B_{\nu]}-B_{\mu \nu}-2 \tilde{m}_{2} f_{[\mu, \nu]}-4 \tilde{m}_{1} C_{\mu \nu}
\end{aligned}
$$

Now let us partially gauge fix such description by settling $f_{\mu}{ }^{a}=0$ and $B_{\mu}=0$. At the same time we solve corresponding algebraic equations $T_{\mu \nu}{ }^{a}=0$ and $\mathcal{B}_{\mu \nu}=0$ :

$$
\Phi_{\mu \nu}^{a}=-\frac{1}{4 m_{1}} \Omega_{[\mu, \nu]}^{a}, \quad C_{\mu \nu}=-\frac{1}{4 \tilde{m}_{1}} B_{\mu \nu}
$$

Then (after some field rescaling) we obtain the following simple Lagrangian for remaining 4 fields:

$$
\begin{aligned}
\mathcal{L}_{0}= & -\frac{3}{4}\left\{\begin{array}{c}
\mu \nu \\
a b
\end{array}\right\} \Omega_{\mu}{ }^{a c d} \Omega_{\nu}{ }^{b c d}-\frac{3}{8}\left\{\begin{array}{c}
\mu \nu \alpha \\
a b c
\end{array}\right\} \Omega_{\mu}{ }^{a b d} D_{\nu} \Omega_{\alpha}{ }^{c d}-\frac{1}{6} C_{a b c}{ }^{2}-\frac{1}{4} e^{\mu}{ }_{a} C^{a b c} D_{\mu} B^{b c}- \\
& -m_{2} e^{\mu}{ }_{a}\left[\frac{3}{2} \Omega_{\mu}{ }^{a b c} B^{b c}+C^{a b c} \Omega_{\mu}{ }^{b c}\right]-\frac{m_{1}{ }^{2}}{2}\left\{\begin{array}{c}
\mu \nu \\
a b
\end{array}\right\} \Omega_{\mu}{ }^{a c} \Omega_{\nu}{ }^{b c}-\frac{\tilde{m}_{1}{ }^{2}}{4} B_{a b}{ }^{2}
\end{aligned}
$$

This Lagrangian is invariant under the following remaining gauge transformations:

$$
\begin{aligned}
\delta \Omega_{\mu}{ }^{a b c} & =D_{\mu} \eta^{a b c}+\frac{4 m_{1}{ }^{2}}{3(d-3)} e_{\mu}{ }^{[a} \eta^{b c]}, \quad \delta \Omega_{\mu}{ }^{a b}=D_{\mu} \eta^{a b}-2 \eta_{\mu}{ }^{a b} \\
\delta C^{a b c} & =6 m_{2} \eta^{a b c}, \quad \delta B^{a b}=-4 m_{2} \eta^{a b}
\end{aligned}
$$


Note that such description turns out to be closely related with Alkalaev-Shaynkman-Vasiliev description for mixed symmetry fields [5] (see also [6, 7]). Indeed gauge fields $\Omega_{\mu}{ }^{a b c}$ and $\Omega_{\mu}{ }^{a b}$ (up to different normalization) correspond to the ASV description of (partially) massless hook in $A d S$, while zero forms $C^{a b c}$ and $B^{a b}$ play the roles of Stueckelberg fields making them massive.

After partial gauge fixing we have 4 gauge invariant objects:

$$
\begin{aligned}
\mathcal{R}_{\mu \nu}{ }^{a b c} & =D_{[\mu} \Omega_{\nu]}{ }^{a b c}+\frac{4 m_{2}}{3(d-3)} e_{[\mu}{ }^{[a} C_{\nu]}{ }^{b c]}+\frac{4 m_{1}{ }^{2}}{3(d-3)} e_{[\mu}{ }^{[a} \Omega_{\nu]}{ }^{b c]} \\
\mathcal{F}_{\mu \nu}{ }^{a b} & =D_{[\mu} \Omega_{\nu]}{ }^{a b}+2 \Omega_{[\mu, \nu]}{ }^{a b}-\frac{2 m_{2}}{(d-3)} e_{[\mu}^{\left[{ }^{[a} B_{\nu]}{ }^{b]}\right.} \\
\mathcal{C}_{\mu}{ }^{a b c} & =D_{\mu} C^{a b c}-6 m_{2} \Omega_{\mu}{ }^{a b c}-\frac{2 m_{1}{ }^{2}}{(d-3)} e_{\mu}{ }^{[a} B^{b c]} \\
\mathcal{B}_{\mu}{ }^{a b} & =D_{\mu} B^{a b}+\frac{4}{3} C_{\mu}{ }^{a b}+4 m_{2} \Omega_{\mu}{ }^{a b}
\end{aligned}
$$

Our next task is to rewrite the free Lagrangian as an expression quadratic in these gauge invariant curvatures. The most general such Lagrangian looks as follows:

$$
\begin{aligned}
\mathcal{L}_{0}= & \left\{\begin{array}{l}
\mu \nu \alpha \beta \\
\left.{ }_{a b d}\right\}
\end{array}\right\}\left[a_{1} \mathcal{R}_{\mu \nu}{ }^{a b e} \mathcal{R}_{\alpha \beta}{ }^{c d e}+a_{2} \mathcal{F}_{\mu \nu}{ }^{a b} \mathcal{F}_{\alpha \beta}{ }^{c d}\right]+\left\{{ }_{a b c}^{\mu \nu \alpha}\right\}\left[a_{5} \mathcal{R}_{\mu \nu}{ }^{a b d} \mathcal{B}_{\alpha}{ }^{c d}+a_{6} \mathcal{F}_{\mu \nu}{ }^{a d} \mathcal{C}_{\alpha}{ }^{b c d}\right]+ \\
& +\left\{{ }_{a b}^{\mu \nu}\right\}\left[a_{3} \mathcal{C}_{\mu}{ }^{a c d} \mathcal{C}_{\nu}{ }^{b c d}+a_{4} \mathcal{B}_{\mu}{ }^{a c} \mathcal{B}_{\nu}{ }^{b c}\right]
\end{aligned}
$$

We have usual differential identities for curvatures:

$$
\begin{aligned}
& D_{[\mu} \mathcal{R}_{\nu \alpha]}{ }^{a b c}=-\frac{4 m_{2}}{3(d-3)} e_{[\mu}^{[a} \mathcal{C}_{\nu, \alpha]}{ }^{b c]}-\frac{4 m_{1}{ }^{2}}{3(d-3)} e_{[\mu}{ }^{[a} \mathcal{F}_{\nu \alpha]}{ }^{b c]} \\
& D_{[\mu} \mathcal{F}_{\nu \alpha]}^{a b}=2 \mathcal{R}_{[\mu \nu, \alpha]}{ }^{a b}+\frac{2 m_{2}}{(d-3)} e_{[\mu}^{\left[{ }^{[a} \mathcal{B}_{\nu, \alpha]}{ }^{b]}\right.} \\
& D_{[\mu} \mathcal{C}_{\nu]}^{a b c}=-6 m_{2} \mathcal{R}_{\mu \nu}{ }^{a b c}+\frac{2 m_{1}{ }^{2}}{(d-3)} e_{[\mu}{ }^{[a} \mathcal{B}_{\nu]}{ }^{b c]} \\
& D_{[\mu} \mathcal{B}_{\nu]}^{a b}=\frac{4}{3} \mathcal{C}_{[\mu, \nu]}^{a b}+4 m_{2} \mathcal{F}_{\mu \nu}^{a b}
\end{aligned}
$$

Note that on the solutions of auxiliary fields $\Omega_{\mu}{ }^{a b c}$ and $C^{a b c}$ we have

$$
\mathcal{F}_{[\mu \nu, \alpha]}^{a}=0, \quad \mathcal{B}_{[\mu, \nu \alpha]}=0 \quad \Longrightarrow \quad \mathcal{R}_{[\mu \nu, \alpha \beta]}^{a}=0
$$

Using these differential identities we can obtain the following 4 identities for curvatures squares:

$$
\begin{aligned}
I_{1}= & \left\{\begin{array}{l}
\mu \nu \alpha \beta \gamma \\
a b c d e
\end{array}\right\} D_{\mu}\left[\mathcal{R}_{\nu \alpha}{ }^{a b c} \mathcal{F}_{\beta \gamma}{ }^{d e}\right]= \\
= & \left\{\begin{array}{l}
\mu \nu \alpha \beta \\
a b c d
\end{array}\right\}\left[3 \mathcal{R}_{\mu \nu}{ }^{a b e} \mathcal{R}_{\alpha \beta}{ }^{c d e}-\frac{2(d-4) m_{1}{ }^{2}}{(d-3)} \mathcal{F}_{\mu \nu}{ }^{a b} \mathcal{F}_{\alpha \beta}{ }^{c d}\right]- \\
& -\frac{2(d-4) m_{2}}{(d-3)}\left\{{ }_{a b c}^{\mu \nu \alpha}\right\}\left[6 \mathcal{R}_{\mu \nu}{ }^{a b d} \mathcal{B}_{\alpha}{ }^{c d}+4 \mathcal{F}_{\mu \nu}{ }^{a d} \mathcal{C}_{\alpha}{ }^{b c d}\right]=0 \\
I_{2}= & \left\{\begin{array}{l}
\mu \nu \alpha \beta \\
{ }_{a b c d}
\end{array}\right\} D_{\mu}\left[\mathcal{R}_{\nu \alpha}{ }^{a b e} \mathcal{C}_{\beta}{ }^{c d e}\right]=
\end{aligned}
$$




$$
\begin{aligned}
& =-3 m_{2}\left\{\begin{array}{c}
{ }^{\mu \nu \alpha \beta} \alpha \\
a b c d
\end{array}\right\} \mathcal{R}_{\mu \nu}{ }^{a b e} \mathcal{R}_{\alpha \beta}{ }^{c d e}-\frac{32(d-4) m_{2}}{3(d-3)}\left\{{ }_{a b}^{\mu \nu}\right\} \mathcal{C}_{\mu}{ }^{a c d} \mathcal{C}_{\nu}{ }^{b c d}+ \\
& +\frac{4(d-4) m_{1}{ }^{2}}{3(d-3)}\left\{{ }_{a b c}^{\mu \nu \alpha}\right\}\left[3 \mathcal{R}_{\mu \nu}{ }^{a b d} \mathcal{B}_{\alpha}{ }^{c d}-2 \mathcal{F}_{\mu \nu}{ }^{a d} \mathcal{C}_{\alpha}{ }^{b c d}\right]=0 \\
& I_{3}=\left\{\begin{array}{c}
\mu \nu \alpha \beta \\
a b c d
\end{array}\right\} D_{\mu}\left[\mathcal{F}_{\nu \alpha}{ }^{a b} \mathcal{B}_{\beta}{ }^{c d}\right]= \\
& =m_{2}\left\{\begin{array}{c}
\mu \nu \alpha \beta \\
a b c d
\end{array}\right\} \mathcal{F}_{\mu \nu}^{a b} \mathcal{F}_{\alpha \beta}{ }^{c d}+\left\{\begin{array}{l}
\mu \nu \alpha \\
a b c
\end{array}\right\}\left[-2 \mathcal{R}_{\mu \nu}{ }^{a b d} \mathcal{B}_{\alpha}{ }^{c d}+\frac{4}{3} \mathcal{F}_{\mu \nu}{ }^{a d} \mathcal{C}_{\alpha}{ }^{b c d}\right]+8 m_{2}\left\{\begin{array}{l}
\mu \nu \\
a b
\end{array}\right\} \mathcal{B}_{\mu}{ }^{a c} \mathcal{B}_{\nu}{ }^{b c}=0 \\
& I_{4}=\left\{\begin{array}{l}
\mu \nu \alpha \\
a b c
\end{array}\right\} D_{\mu}\left[\mathcal{C}_{\nu}^{a b d} \mathcal{B}_{\alpha}{ }^{c d}\right]= \\
& =\left\{{ }_{a b c}^{\mu \nu \alpha}\right\}\left[-3 m_{2} \mathcal{R}_{\mu \nu}{ }^{a b d} \mathcal{B}_{\alpha}{ }^{c d}-2 m_{2} \mathcal{F}_{\mu \nu}{ }^{a d} \mathcal{C}_{\alpha}{ }^{b c d}\right]+\left\{{ }_{a b}^{\mu \nu}\right\}\left[-\frac{8}{3} \mathcal{C}_{\mu}{ }^{a c d} \mathcal{C}_{\nu}{ }^{b c d}+4 m_{1}{ }^{2} \mathcal{B}_{\mu}{ }^{a c} \mathcal{B}_{\nu}{ }^{b c}\right]=0
\end{aligned}
$$

These 4 identities are not independent. Indeed by direct calculations one can show:

$$
m_{2} X_{1}+X_{2}+\frac{2(d-4) m_{1}^{2}}{(d-3)} X_{3}-\frac{4(d-4) m_{2}}{(d-3)} X_{4}=0
$$

Thus we have 3 independent identities so if we require that the Lagrangian quadratic in curvatures correctly reproduce free Lagrangian for massive hook given above we would expect that we obtain solution with 3 arbitrary parameters. This turns out to be the case. Note however that one has to be careful using this freedom because as our previous experience shows switching on an interaction tends to partially resolve this ambiguity. We will use the following simple choice for free Lagrangian:

$$
\begin{aligned}
& \mathcal{L}_{0}=\left\{\begin{array}{c}
\mu \nu \alpha \beta \\
{ }_{a b c d}
\end{array}\right\}\left[a_{1} \mathcal{R}_{\mu \nu}{ }^{a b e} \mathcal{R}_{\alpha \beta}{ }^{c d e}+a_{2} \mathcal{F}_{\mu \nu}{ }^{a b} \mathcal{F}_{\alpha \beta}{ }^{c d}\right]+\left\{{ }_{a b}^{\mu \nu}\right\}\left[a_{3} \mathcal{C}_{\mu}{ }^{a c d} \mathcal{C}_{\nu}{ }^{b c d}+a_{4} \mathcal{B}_{\mu}{ }^{a c} \mathcal{B}_{\nu}{ }^{b c}\right] \\
& a_{1}=-\frac{9}{512 m_{1}^{2}}, \quad a_{2}=-\frac{3}{256(d-3)}, \quad a_{3}=-\frac{(d-4)}{16 m_{1}^{2}(d-3)}, \quad a_{4}=-\frac{3}{32(d-3)}
\end{aligned}
$$

Later on we will see that such choice is compatible with the possibility to switch on an interaction.

\section{Cubic gravitational interactions}

For gravitational field we will use notations $h_{\mu}{ }^{a}$ and $\omega_{\mu}^{a b}$. Gauge transformations for the free massless field in $(A) d S$ space have the form:

$$
\delta_{0} h_{\mu}^{a}=D_{\mu} \chi^{a}+\chi_{\mu}{ }^{a}, \quad \delta_{0} \omega_{\mu}^{a b}=D_{\mu} \chi^{a b}+\kappa e_{\mu}{ }^{[a} \chi^{b]}
$$

Correspondingly we have two gauge invariant objects (linearized curvature and torsion):

$$
\begin{aligned}
R_{\mu \nu}{ }^{a b} & =D_{[\mu} \omega_{\nu]}^{a b}+\kappa e_{[\mu}^{[a} h_{\nu]}^{b]} \\
T_{\mu \nu}{ }^{a} & =D_{[\mu} h_{\nu]}{ }^{a}-\omega_{[\mu, \nu]}{ }^{a}
\end{aligned}
$$

For non-zero values of cosmological constant the free Lagrangian can be written as follows:

$$
\mathcal{L}_{0}=-\frac{1}{32 \kappa(d-3)}\left\{\begin{array}{c}
\mu \nu \alpha \beta \\
a b c d
\end{array}\right\} R_{\mu \nu}^{a b} R_{\alpha \beta}{ }^{c d}
$$


According to general procedure our first task is to find deformations for all gauge invariant curvatures supplemented with appropriate corrections to gauge transformations such that variations of these deformed curvatures were proportional to the free ones.

Deformations for hooks curvatures. Let us consider deformations for hooks curvatures corresponding to minimal gravitational interactions:

$$
\begin{aligned}
\Delta \mathcal{R}_{\mu \nu}{ }^{a b c} & =\omega_{[\mu}{ }^{d[a} \Omega_{\nu]}{ }^{b c] d}-\frac{4 m_{2}}{3(d-3)}\left[h_{[\mu}{ }^{[a} C_{\nu]}{ }^{b c]}+e_{[\mu}{ }^{[a} C^{b c] d} h_{\nu]}{ }^{d}\right]-\frac{4 m_{1}{ }^{2}}{3(d-3)} h_{[\mu}{ }^{[a} \Omega_{\nu]}{ }^{b c]} \\
\Delta \mathcal{F}_{\mu \nu}{ }^{a b} & =-\omega_{[\mu}{ }^{c[a} \Omega_{\nu]}{ }^{b] c}-2 \Omega_{[\mu}{ }^{a b c} h_{\nu]}{ }^{c}+\frac{2 m_{2}}{(d-3)}\left[h_{[\mu}{ }^{[a} B_{\nu]}{ }^{b]}-e_{[\mu}{ }^{[a} B^{b] c} h_{\nu]}{ }^{c}\right] \\
\Delta \mathcal{C}_{\mu}{ }^{a b c} & =\omega_{[\mu}{ }^{d[a} C^{b c] d}+\frac{2 m_{1}{ }^{2}}{(d-3)} h_{\mu}{ }^{[a} B^{b c]}, \quad \Delta \mathcal{B}_{\mu}{ }^{a b}=-\omega_{\mu}{ }^{c[a} B^{b] c}-\frac{4}{3} C^{a b c} h_{\mu}{ }^{c}
\end{aligned}
$$

Similarly, the appropriate corrections to gauge transformations turn out to be:

$$
\begin{aligned}
\delta \Omega_{\mu}{ }^{a b c}= & -\chi^{d[a} \Omega_{\mu}{ }^{b c] d}+\frac{4 m_{2}}{3(d-3)}\left[C_{\mu}{ }^{[a b} \chi^{c]}+e_{\mu}{ }^{[a} C^{b c] d} \chi^{d}\right]+\frac{4 m_{1}{ }^{2}}{3(d-3)} \Omega_{\mu}{ }^{[a b} \chi^{c]}+ \\
& +\omega_{\mu}{ }^{d[a} \eta^{b c] d}-\frac{4 m_{1}{ }^{2}}{3(d-3)} h_{\mu}{ }^{[a} \eta^{b c]} \\
\delta \Omega_{\mu}{ }^{a b}= & \chi^{c[a} \Omega_{\mu}{ }^{b] c}-2 \Omega_{\mu}{ }^{a b c} \chi^{c}+\frac{2 m_{2}}{(d-3)}\left[B_{\mu}{ }^{[a} \chi^{b]}-e_{\mu}{ }^{[a} B^{b] c} \chi^{c}\right]-\omega_{\mu}{ }^{c[a} \eta^{b] c}+2 \eta^{a b c} h_{\mu}{ }^{c} \\
\delta C^{a b c}= & -\chi^{d[a} C^{b c] d}-\frac{2 m_{1}{ }^{2}}{(d-3)} B^{[a b} \chi^{c]}, \quad \delta B^{a b}=\chi^{c[a} B^{b] c}+\frac{4}{3} C^{a b c} \chi^{c}
\end{aligned}
$$

Taking into account these corrections we obtain the following transformations of deformed curvatures under the hook's $\eta^{a b c}$ and $\eta^{a b}$ transformations:

$$
\begin{aligned}
\delta \hat{\mathcal{R}}_{\mu \nu}{ }^{a b c} & =R_{\mu \nu}{ }^{d[a} \eta^{b c] d}-\frac{4 m_{1}{ }^{2}}{3(d-3)} T_{\mu \nu}{ }^{[a} \eta^{b c]} \\
\delta \hat{\mathcal{F}}_{\mu \nu}{ }^{a b} & =2 \eta^{a b c} T_{\mu \nu}{ }^{c}-R_{\mu \nu}{ }^{c[a} \eta^{b] c}
\end{aligned}
$$

Deformations of gravitational curvatures. The most general ansatz for such deformations quadratic in fields looks like (schematically)1:

$$
\begin{aligned}
& \hat{R} \sim R \oplus \Omega_{3} \Omega_{3} \oplus \Omega_{3} B \oplus B B \oplus \Omega_{2} \Omega_{2} \oplus \Omega_{2} C \oplus C C \\
& \hat{T} \sim T \oplus \Omega_{3} \Omega_{2} \oplus \Omega_{3} C \oplus \Omega_{2} B \oplus C B
\end{aligned}
$$

where $\Omega_{3}$ stands for $\Omega_{\mu}^{a b c}$ and $\Omega_{2}-$ for $\Omega_{\mu}^{a b}$. Due to the presence of zero forms $C^{a b c}$ and $B^{a b}$ there exists a possibility to make field redefinitions of the form:

$$
\begin{aligned}
\omega_{\mu}{ }^{a b} & \Rightarrow \omega_{\mu}{ }^{a b}+\kappa_{1} C^{a b c} B_{\mu}{ }^{c}+\kappa_{2} C_{\mu}{ }^{c[a} B^{b] c}+\kappa_{3} e_{\mu}{ }^{[a} C^{b] c d} B^{c d} \\
h_{\mu}{ }^{a} & \Rightarrow h_{\mu}{ }^{a}+\kappa_{4} C^{a b c} C_{\mu}{ }^{b c}+\kappa_{5} e_{\mu}{ }^{a} C^{b c d} C^{b c d}+\kappa_{6} B^{a b} B_{\mu}{ }^{b}+\kappa_{7} e_{\mu}{ }^{a} B^{b c} B^{b c}
\end{aligned}
$$

\footnotetext{
${ }^{1}$ Really we have considered the general case without partial gauge fixing where all eight fields are present. In this, the resulting expressions for deformed curvatures contain auxiliary fields $\Omega_{3}, \Omega_{2}, C$ and $B$ only. Thus, at least in this particular case, partial gauge fixing "commute" with switching on interactions and we may use it to simplify calculations without any lost of generality.
} 
which we will use to simplify all subsequent expressions 2 . In this, the resulting expressions for curvatures can be casted into the form:

$$
\begin{aligned}
& \hat{R}_{\mu \nu}{ }^{a b}=R_{\mu \nu}^{a b}+a_{0}\left[\Omega_{[\mu}^{a c d} \Omega_{\nu]}^{b c d}+\frac{m_{2}}{(d-3)} e_{[\mu}{ }^{[a} \Omega_{\nu]}{ }^{b] c d} B^{c d}+\frac{4}{9(d-3)} C_{[\mu}^{c a} C_{\nu]}^{b c}-\right. \\
& \left.-\frac{4 m_{1}{ }^{2}}{3(d-3)} \Omega_{[\mu}{ }^{c a} \Omega_{\nu]}{ }^{b c}-\frac{m_{1}{ }^{2}}{3(d-3)^{2}} B_{[\mu}^{a} B_{\nu]}^{b}+\frac{m_{1}{ }^{2}}{3(d-3)^{2}} e_{[\mu}^{a} e_{\nu]}^{b} B^{c d} B^{c d}\right] \\
& \hat{T}_{\mu \nu}^{a}=T_{\mu \nu}^{a}+a_{0}\left[\frac{1}{2} \Omega_{[\mu}^{a b c} \Omega_{\nu]}^{b c}-\frac{1}{(d-3)} C_{[\mu}^{a b} B_{\nu]}^{b}-\frac{1}{6(d-3)} e_{[\mu}^{a} C_{\nu]}^{b c} B^{b c}\right]
\end{aligned}
$$

while appropriate corrections to gauge transformations take the form:

$$
\begin{aligned}
\delta \omega_{\mu}{ }^{a b} & =-a_{0} \eta^{c d[a} \Omega_{\mu}{ }^{b] c d}+\frac{m_{2} a_{0}}{(d-3)} e_{\mu}{ }^{[a} \eta^{b] c d} B^{c d}+\frac{4 m_{1}{ }^{2} a_{0}}{3(d-3)} \eta^{c[a} \Omega_{\mu}{ }^{b] c} \\
\delta h_{\mu}{ }^{a} & =\frac{a_{0}}{2}\left[\Omega_{\mu}{ }^{a b c} \eta^{b c}-\eta^{a b c} \Omega_{\mu}{ }^{b c}\right]
\end{aligned}
$$

For what follows we will need transformations for deformed Riemann tensor under the hook's $\eta^{a b c}$ and $\eta^{a b}$ transformations:

$$
\delta \hat{R}_{\mu \nu}{ }^{a b}=-a_{0} \eta^{c d[a} \mathcal{R}_{\mu \nu}{ }^{b] c d}+\frac{4 m_{1}^{2} a_{0}}{3(d-3)} \eta^{c[a} \mathcal{F}_{\mu \nu}{ }^{b] c}
$$

Gravitational interaction. Now according to general procedure we consider the sum of free Lagrangians for hook and graviton where all curvatures are replaced with the deformed ones:

$$
\begin{aligned}
\mathcal{L}_{0}= & \left\{\begin{array}{l}
\mu \nu \alpha \beta \\
a b c d
\end{array}\right\}\left[a_{1} \hat{\mathcal{R}}_{\mu \nu}{ }^{a b e} \hat{\mathcal{R}}_{\alpha \beta}{ }^{c d e}+a_{2} \hat{\mathcal{F}}_{\mu \nu}{ }^{a b} \hat{\mathcal{F}}_{\alpha \beta}{ }^{c d}\right]+\left\{\begin{array}{c}
\mu \nu \\
a b
\end{array}\right\}\left[a_{3} \hat{\mathcal{C}}_{\mu}{ }^{a c d} \hat{\mathcal{C}}_{\nu}{ }^{b c d}+a_{4} \hat{\mathcal{B}}_{\mu}{ }^{a c} \hat{\mathcal{B}}_{\nu}{ }^{b c}\right]- \\
& -\frac{1}{32 \kappa(d-3)}\left\{\begin{array}{l}
\mu \nu \alpha \beta \\
a b c d
\end{array}\right\} \hat{R}_{\mu \nu}{ }^{a b} \hat{R}_{\alpha \beta}{ }^{c d}
\end{aligned}
$$

Now we have to consider all variations that do not vanish on shell and try to adjust coefficients so that all them vanish. Transformations for hook curvatures we have to take care on look like:

$$
\delta \hat{\mathcal{R}}_{\mu \nu}{ }^{a b c}=R_{\mu \nu}{ }^{d[a} \eta^{b c] d}, \quad \delta \hat{\mathcal{F}}_{\mu \nu}{ }^{a b}=-R_{\mu \nu}{ }^{c[a} \eta^{b] c}
$$

while for deformed Riemann tensor they are given in (23).

Variations under the $\eta^{a b c}$ transformations give us:

$$
-\left\{\begin{array}{l}
\mu \nu \alpha \beta \\
a b c d
\end{array}\right\}\left[4 a_{1} \mathcal{R}_{\mu \nu}{ }^{a b e} R_{\alpha \beta}{ }^{c f} \eta^{d e f}+\frac{a_{0}}{8 \kappa(d-3)} \mathcal{R}_{\mu \nu}{ }^{a e f} R_{\alpha \beta}{ }^{b c} \eta^{d e f}\right]
$$

Using on shell identities $\mathcal{R}_{[\mu \nu, \alpha \beta]}^{a}=0$ and $R_{[\mu \nu, \alpha]}^{a}=0$ one can show that the following identity holds:

$$
\left\{\begin{array}{c}
\mu \nu \alpha \beta \\
a b c d
\end{array}\right\}\left[2 \mathcal{R}_{\mu \nu}^{a b e} R_{\alpha \beta}{ }^{c f}-\mathcal{R}_{\mu \nu}{ }^{a e f} R_{\alpha \beta}{ }^{b c}\right] \eta^{d e f}=0
$$

\footnotetext{
${ }^{2}$ Note that the choice we make here has to be in agreement with choice for the parameters in the free Lagrangian. As we will see later on our choices are indeed consistent.
} 
Thus we have to put:

$$
a_{1}=-\frac{a_{0}}{16 \kappa(d-3)}
$$

Similarly, variations under the $\eta^{a b}$ transformations produce:

$$
\left\{\begin{array}{c}
\mu \nu \alpha \beta \\
a b c d
\end{array}\right\}\left[4 a_{2} \mathcal{F}_{\mu \nu}{ }^{a b} R_{\alpha \beta}{ }^{c e} \eta^{d e}-\frac{m_{1}{ }^{2} a_{0}}{6 \kappa(d-3)^{2}} \mathcal{F}_{\mu \nu}{ }^{a e} R_{\alpha \beta}{ }^{b c} \eta^{d e}\right]
$$

Again using on shell identities $\mathcal{F}_{[\mu \nu, \alpha]}^{a}=0$ and $R_{[\mu \nu, \alpha]}^{a}=0$ one can show that the following identity holds:

$$
\left\{\begin{array}{c}
\mu \nu \alpha \beta \\
a b c d
\end{array}\right\}\left[\mathcal{F}_{\mu \nu}^{a b} R_{\alpha \beta}{ }^{c e}+\mathcal{F}_{\mu \nu}{ }^{a e} R_{\alpha \beta}{ }^{b c}\right] \eta^{d e}=0
$$

Thus we obtain:

$$
a_{2}=-\frac{m_{1}{ }^{2} a_{0}}{24 \kappa(d-3)^{2}}=\frac{2 m_{1}^{2} a_{1}}{3(d-3)}
$$

Note that the resulting relation for $a_{1}$ and $a_{2}$ is in agreement with our choice for free Lagrangian.

Thus the Lagrangian (24) with the deformed curvatures defined in (18) and (22) gives us a correct set of cubic gravitational vertices including standard minimal interactions together with non-minimal higher derivatives ones. There are two particular limits that one can consider here. First of all we may take $m_{2}=0$. In this limit Stueckelberg fields $C^{a b c}$ and $B^{a b}$ completely decouple and the result (up to different field normalization) completely agree with the results obtained previously in [1].

Other interesting and important limit is a flat limit $\kappa \rightarrow 0$ (i.e. $m_{2}^{2} \rightarrow m_{1}^{2} / 3$ ). The peculiarity here is related with the fact that for massless graviton it is possible to rewrite Lagrangian as an expression quadratic in curvatures for non-zero values of cosmological constant only. But from the relation (26) we obtain:

$$
a_{0}=\frac{9(d-3) \kappa}{32 m_{1}^{2}}
$$

so that at least in the linear approximation the contribution from gravity part of the Lagrangian is non-singular in a flat limit.

\section{Conclusion}

Thus we have seen that Fradkin-Vasiliev approach together with frame-like gauge invariant formalism for massive fields allows one effectively investigate possible interactions for massive and/or massless fields. The massive hook (as well as massive spin 2) is one of the simplest examples possible but it is clear that such approach can be applied to higher spin fields (both symmetric and mixed symmetry ones) as well. One of the questions that deserves further study is the problem of flat limit for gravitational interactions. The reason is that the Lagrangian for massless graviton can be written as square of curvature for the non-zero cosmological constant only though as we have seen in the linear approximation flat limit is non-singular. Also it would be interesting to understand a striking difference 
between massless representations in $A d S$ and $d S$ spaces as far as switching on interactions is concerned.

\section{Acknowledgment}

Author is grateful to N. Boulanger, E. Skvortsov and M. Vasiliev for many useful discussions. The work was supported in parts by RFBR grant No.11-02-00814.

\section{A Partially massless case in a de Sitter space}

Here we will try to switch on gravitational interactions for (partially) massless mixed symmetry field in $d S$ space corresponding ; to $m_{1} \rightarrow 0$ limit.

Kinematics. For convenience we reproduce here gauge transformations for this case:

$$
\begin{aligned}
\delta_{0} \Phi_{\mu \nu}^{a} & =D_{[\mu} z_{\nu]}^{a}+\eta_{\mu \nu}^{a}+\frac{4 m_{2}}{(d-3)} e_{[\mu}^{a} \zeta_{\nu]}, \quad \delta_{0} \Omega_{\mu}{ }^{a b c}=D_{\mu} \eta^{a b c} \\
\delta_{0} C_{\mu \nu} & =D_{[\mu} \zeta_{\nu]}-2 m_{2} z_{[\mu \nu]}, \quad \delta_{0} C^{a b c}=6 m_{2} \eta^{a b c}
\end{aligned}
$$

Here $8 m_{2}^{2}=(d-3) \kappa$. Correspondingly we have four gauge invariant objects:

$$
\begin{aligned}
\mathcal{R}_{\mu \nu}{ }^{a b c} & =D_{[\mu} \Omega_{\nu]}^{a b c}+\frac{4 m_{2}}{3(d-3)} e_{[\mu}^{[a} C_{\nu]}{ }^{b c]} \\
\mathcal{T}_{\mu \nu \alpha}{ }^{a} & =D_{[\mu} \Phi_{\nu \alpha]}{ }^{a}-\Omega_{[\mu, \nu \alpha]}{ }^{a}+\frac{4 m_{2}}{(d-3)} e_{[\mu}{ }^{a} C_{\nu \alpha]} \\
\mathcal{C}_{\mu}{ }^{a b c} & =D_{\mu} C^{a b c}-6 m_{2} \Omega_{\mu}{ }^{a b c} \\
\mathcal{C}_{\mu \nu \alpha} & =D_{[\mu} C_{\nu \alpha]}-C_{\mu \nu \alpha}+2 m_{2} \Phi_{[\mu \nu, \alpha]}
\end{aligned}
$$

It is not hard to express the free Lagrangian in terms of these gauge invariant curvatures:

$$
\begin{aligned}
& \mathcal{L}_{0}=\left\{\begin{array}{c}
{ }_{a b c d} \nu \beta \\
a b c d
\end{array}\left[a_{1} \mathcal{R}_{\mu \nu}{ }^{a b e} \mathcal{R}_{\alpha \beta}{ }^{c d e}+a_{2}\left\{{ }_{a b}^{\mu \nu}\right\} \mathcal{C}_{\mu}{ }^{a c d} \mathcal{C}_{\nu}{ }^{b c d}+a_{3} \mathcal{T}_{\mu \nu \alpha}{ }^{a} \mathcal{C}_{\beta}{ }^{b c d}\right]\right. \\
& \frac{512 m^{2}(d-4) a_{1}}{3(d-3)}-48 m^{2} a_{2}=-1, \quad a_{3}=-\frac{1}{72 m}
\end{aligned}
$$

Again there is an ambiguity in the choice of parameters related with differential identities. Indeed we have:

$$
\begin{aligned}
D_{[\mu} \mathcal{R}_{\nu \alpha]}{ }^{a b c} & =-\frac{4 m_{2}}{3(d-3)} e_{[\mu}{ }^{[a} \mathcal{C}_{\nu, \alpha]}{ }^{b c]} \\
D_{[\mu} \mathcal{C}_{\nu]}{ }^{a b c} & =-6 m_{2} \mathcal{R}_{\mu \nu}{ }^{a b c}
\end{aligned}
$$

As a result we obtain:

$$
\left\{\begin{array}{c}
{ }_{a \nu \alpha \beta}{ }_{a b c d}
\end{array}\right\} D_{\mu}\left[\mathcal{R}_{\nu \alpha}{ }^{a b e} \mathcal{C}_{\beta}{ }^{c d e}\right]=-3 m_{2}\left[\left\{\begin{array}{c}
\mu \nu \alpha \beta \\
a b c d
\end{array}\right\} \mathcal{R}_{\mu \nu}{ }^{a b e} \mathcal{R}_{\alpha \beta}{ }^{c d e}+\frac{32(d-4)}{9(d-3)}\left\{{ }_{a b}^{\mu \nu}\right\} \mathcal{C}_{\mu}{ }^{a c d} \mathcal{C}_{\nu}{ }^{b c d}\right]=0
$$


Deformations for gravitational curvatures. This time, due to the presence of zero form, there is an ambiguity related with possible field redefinitions, namely:

$$
h_{\mu}{ }^{a} \Longrightarrow h_{\mu}{ }^{a}+\alpha_{1} C_{\mu}{ }^{b c} C^{a b c}+\alpha_{2} e_{\mu}{ }^{a} C^{b c d} C^{b c d}
$$

Using these redefinitions deformed curvatures can be casted into the form:

$$
\begin{aligned}
\hat{R}_{\mu \nu}{ }^{a b} & =D_{[\mu} \omega_{\nu]}^{a b}+\kappa e_{[\mu}^{[a} h_{\nu]}^{b]}+6 m_{2} a_{0}\left[\Omega_{[\mu}{ }^{a c d} \Omega_{\nu]}{ }^{b c d}-\frac{4}{3(d-3)} C_{[\mu}{ }^{a c} C_{\nu]}{ }^{b c}\right] \\
\hat{T}_{\mu \nu}{ }^{a} & =D_{[\mu} h_{\nu]}^{a}-\omega_{[\mu, \nu]}{ }^{a}-a_{0} \Omega_{[\mu}{ }^{a b c} C_{\nu]}{ }^{b c}
\end{aligned}
$$

In this, appropriate corrections to gauge transformations look as follows:

$$
\delta \omega_{\mu}^{a b}=-6 m_{2} a_{0} \eta^{c d[a} \Omega_{\mu}{ }^{b] c d}, \quad \delta h_{\mu}{ }^{a}=a_{0} \eta^{a b c} C_{\mu}{ }^{b c}
$$

Taking into account these corrections we obtain the following transformations for deformed curvatures:

$$
\delta \hat{R}_{\mu \nu}{ }^{a b}=-6 m_{2} a_{0} \eta^{c d[a} \mathcal{R}_{\mu \nu}{ }^{b] c d}, \quad \delta \hat{T}_{\mu \nu}{ }^{a}=a_{0} \eta^{a b c} \mathcal{C}_{[\mu, \nu]}^{b c}
$$

Deformations for hook's curvatures. In these case the desired results can be easily obtained by the usual substitutions corresponding to minimal gravitational interactions:

$$
\begin{aligned}
\hat{\mathcal{R}}_{\mu \nu}{ }^{a b c}= & D_{[\mu} \Omega_{\nu]}{ }^{a b c}+\frac{4 m_{2}}{3(d-3)} e_{[\mu}{ }^{[a} C_{\nu]}{ }^{b c]}+ \\
& +\Omega_{[\mu}{ }^{d[a b} \omega_{\nu]}{ }^{c] d}-\frac{4 m_{2}}{3(d-3)} h_{[\mu}{ }^{a} C_{\nu]}{ }^{b c]}-\frac{4 m_{2}}{3(d-3)} e_{[\mu}{ }^{[a} C^{b c]} h_{\nu]}{ }^{d} \\
\hat{\mathcal{T}}_{\mu \nu \alpha}{ }^{a}= & D_{[\mu} \Phi_{\nu \alpha]}{ }^{a}-\Omega_{[\mu, \nu \alpha]}{ }^{a}+\frac{4 m_{2}}{(d-3)} e_{[\mu}{ }^{a} C_{\nu \alpha]}- \\
& -\omega_{[\mu}{ }^{a b} \Phi_{\nu \alpha]}{ }^{b}-\Omega_{[\mu, \nu}{ }^{a b} h_{\alpha]}{ }^{b}-\frac{4 m_{2}}{(d-3)} h_{[\mu}{ }^{a} C_{\nu \alpha]} \\
\hat{\mathcal{C}}_{\mu}{ }^{a b c}= & D_{\mu} C^{a b c}-6 m_{2} \Omega_{\mu}{ }^{a b c}+\omega_{\mu}{ }^{d[a} C^{b c] d} \\
\hat{\mathcal{C}}_{\mu \nu \alpha}= & D_{[\mu} C_{\nu \alpha]}-C_{\mu \nu \alpha}+2 m_{2} \Phi_{[\mu \nu, \alpha]}+h_{[\mu}{ }^{a} C_{\nu \alpha]}{ }^{a}-2 m_{2} \Phi_{[\mu \nu}{ }^{a} h_{\alpha]}{ }^{a}
\end{aligned}
$$

Corrections to gauge transformations turn out to be:

$$
\begin{aligned}
\delta \Omega_{\mu}{ }^{a b c} & =-\chi^{d[a} \Omega_{\mu}{ }^{b c] d}+\frac{4 m_{2}}{3(d-3)}\left[\chi^{[a} C_{\mu}{ }^{b c]}-e_{\mu}{ }^{[a} C^{b c] d} \chi^{d}\right]-\eta^{d[a b} \omega_{\mu}{ }^{c] d} \\
\delta \Phi_{\mu \nu}{ }^{a} & =\chi^{a b} \Phi_{\mu \nu}{ }^{b}+\Omega_{[\mu, \nu]}{ }^{a b} \chi^{b}+\frac{4 m_{2}}{(d-3)} \chi^{a} C_{\mu \nu}+\eta_{[\mu}{ }^{a b} h_{\nu]}{ }^{b}-\omega_{[\mu}{ }^{a b} z_{\nu]}{ }^{b}-\frac{4 m_{2}}{(d-3)} h_{[\mu}{ }^{a} \zeta_{\nu]} \\
\delta C^{a b c} & =-\chi^{d[a} C^{b c] d}, \quad \delta C_{\mu \nu}=-\chi^{a} C_{\mu \nu}{ }^{a}+2 m_{2} \Phi_{\mu \nu}{ }^{a} \chi^{a}+2 m_{2} z_{[\mu}{ }^{a} h_{\nu]}{ }^{a}
\end{aligned}
$$

Under the hook's gauge transformations $\eta^{a b c}, z_{\mu}{ }^{a}$ and $\zeta_{\mu}$ these deformed curvatures transform as follows:

$$
\begin{aligned}
\delta \hat{\mathcal{R}}_{\mu \nu}{ }^{a b c} & =-\eta^{d[a b} R_{\mu \nu}{ }^{c] d}, \quad \delta \hat{\mathcal{C}}_{\mu \nu \alpha}=-2 m_{2} T_{[\mu \nu}{ }^{a} z_{\alpha]}{ }^{a} \\
\delta \hat{\mathcal{T}}_{\mu \nu \alpha}{ }^{a} & =-\eta_{[\mu}{ }^{a b} T_{\nu \alpha]}{ }^{b}-R_{[\mu \nu}{ }^{a b} z_{\alpha]}{ }^{b}-\frac{4 m_{2}}{3(d-3)} T_{[\mu \nu}{ }^{a} \zeta_{\alpha]}
\end{aligned}
$$


Gravitational interactions. Following general procedure we consider sum of the free Lagrangians for hook and graviton but with all curvatures replaced with the deformed ones:

$$
\begin{aligned}
\mathcal{L}_{0}= & \left\{\begin{array}{c}
{ }^{\mu \nu \alpha \beta} \\
a b c d
\end{array}\right\}\left[a_{1} \hat{\mathcal{R}}_{\mu \nu}{ }^{a b e} \hat{\mathcal{R}}_{\alpha \beta}{ }^{c d e}+a_{2}\left\{\begin{array}{l}
\mu \nu \\
a b
\end{array}\right\} \hat{\mathcal{C}}_{\mu}{ }^{a c d} \hat{\mathcal{C}}_{\nu}{ }^{b c d}+a_{3} \hat{\mathcal{T}}_{\mu \nu \alpha}{ }^{a} \hat{\mathcal{C}}_{\beta}{ }^{b c d}\right]- \\
& -\frac{1}{256 m_{2}{ }^{2}}\left\{\begin{array}{c}
\mu \nu \alpha \beta \\
a b c d
\end{array}\right\} \hat{R}_{\mu \nu}{ }^{a b} \hat{R}_{\alpha \beta}{ }^{c d}
\end{aligned}
$$

Now we have to take care on variations that do not vanish on shell:

$$
\delta \hat{R}_{\mu \nu}^{a b}=-6 m_{2} a_{0} \eta^{c d[a} \mathcal{R}_{\mu \nu}{ }^{b] c d}, \quad \delta \hat{\mathcal{R}}_{\mu \nu}{ }^{a b c}=-\eta^{d[a b} R_{\mu \nu}{ }^{c] d}, \quad \delta \hat{\mathcal{T}}_{\mu \nu \alpha}{ }^{a}=-R_{[\mu \nu}^{a b} z_{\alpha]}{ }^{b}
$$

and try to adjust coefficients so that all of them vanish. It is easy to see that this time it is impossible. Crucial point $-z_{\mu}{ }^{a}$ transformations that give

$$
\delta \mathcal{L} \sim\left\{\begin{array}{c}
\mu \nu \alpha \beta \\
a b c d
\end{array}\right\} \mathcal{C}_{\mu}^{a b c} R_{\nu \alpha}{ }^{d e} z_{\beta}{ }^{e}
$$

and this can not be compensated even on mass shell!

\section{References}

[1] N. Boulanger, E. D. Skvortsov, Yu. M. Zinoviev "Gravitational cubic interactions for a simple mixed-symmetry gauge field in AdS and flat backgrounds", arXiv:1107.1872.

[2] Yu. M. Zinoviev "Spin 3 cubic vertices in a frame-like formalism", JHEP 08 (2010) 084, arXiv:1007.0158.

[3] E. S. Fradkin, M. A. Vasiliev "On the gravitational interaction of massless higher-spin fields", Phys. Lett. B189 (1987) 89.

[4] E. S. Fradkin, M. A. Vasiliev "Cubic interaction in extended theories of massless higherspin fields", Nucl. Phys. B291 (1987) 141.

[5] K. B. Alkalaev, O. V. Shaynkman, M. A. Vasiliev "On the Frame-Like Formulation of Mixed-Symmetry Massless Fields in (A)dS(d)", Nucl. Phys. B692 (2004) 363, arXiv:hep-th/0311164.

[6] K. B. Alkalaev, O. V. Shaynkman, M. A. Vasiliev "Lagrangian Formulation for Free Mixed-Symmetry Bosonic Gauge Fields in (A)dS(d)", JHEP 0508 (2005) 069, arXiv:hep-th/0501108.

[7] K. B. Alkalaev, O. V. Shaynkman, M. A. Vasiliev "Frame-like formulation for free mixed-symmetry bosonic massless higher-spin fields in AdS(d)", arXiv:hep-th/0601225.

[8] Yu. M. Zinoviev "First Order Formalism for Massive Mixed Symmetry Tensor Fields in Minkowski and (A)dS Spaces", arXiv:hep-th/0306292.

[9] Yu. M. Zinoviev "Towards frame-like gauge invariant formulation for massive mixed symmetry bosonic fields", Nucl. Phys. B812 (2009) 46, arXiv:0809.3287. 
[10] G. Barnich, M. Henneaux "Consistent couplings between fields with a gauge freedom and deformations of the master equations", Phys. Lett. B311 (1993) 123, arXiv:hep-th/9304057.

[11] M. Henneaux "Consistent interactions between gauge fields: The cohomological approach", Contemp. Math. 219 (1998) 93, arXiv:hep-th/9712226.

[12] M. A. Vasiliev "Cubic Interactions of Bosonic Higher Spin Gauge Fields in $A d S_{5}$ ", Nucl.Phys. B616 (2001) 106-162; Erratum-ibid. B652 (2003) 407, arXiv:hep-th/0106200.

[13] K. B. Alkalaev, M. A. Vasiliev "N=1 Supersymmetric Theory of Higher Spin Gauge Fields in AdS(5) at the Cubic Level", Nucl.Phys. B655 (2003) 57-92, arXiv:hep-th/0206068.

[14] K.B. Alkalaev "FV-type action for AdS(5) mixed-symmetry fields", JHEP 1103 (2011) 031, arXiv:1011.6109.

[15] M. A. Vasiliev "'Gauge' form of description of massless fields with arbitrary spin", Sov. J. Nucl. Phys. 32 (1980) 439.

[16] V. E. Lopatin, M. A. Vasiliev "Free massless bosonic fields of arbitrary spin in ddimensional de sitter space", Mod. Phys. Lett. A3 (1988) 257.

[17] M. A. Vasiliev "Free massless fermionic fields of arbitrary spin in d-dimensional de sitter space", Nucl. Phys. B301 (1988) 26.

[18] Yu. M. Zinoviev "Frame-like gauge invariant formulation for massive high spin particles", Nucl. Phys. B808 (2009) 185, arXiv:0808.1778.

[19] D. S. Ponomarev, M. A. Vasiliev "Frame-Like Action and Unfolded Formulation for Massive Higher-Spin Fields", Nucl. Phys. B839 (2010) 466, arXiv:1001.0062.

[20] Yu. M. Zinoviev "Frame-like gauge invariant formulation for mixed symmetry fermionic fields", Nucl. Phys. B821 (2009) 21-47, arXiv:0904.0549.

[21] Yu. M. Zinoviev "Towards frame-like gauge invariant formulation for massive mixed symmetry bosonic fields. II. General Young tableau with two rows", Nucl. Phys. B826 (2010) 490, arXiv:0907.2140.

[22] Yu. M. Zinoviev "On electromagnetic interactions for massive mixed symmetry field", JHEP 03 (2011) 082, arXiv:1012.2706.

[23] L. Brink, R. R. Metsaev, M. A. Vasiliev "How massless are massless fields in AdS $S_{d}$, Nucl. Phys. B586 (2000) 183, arXiv:hep-th/0005136.

[24] N. Boulanger, C. Iazeolla, P. Sundell "Unfolding Mixed-Symmetry Fields in AdS and the BMV Conjecture: I. General Formalism", JHEP 0907 (2009) 013, arXiv:0812.3615.

[25] N. Boulanger, C. Iazeolla, P. Sundell "Unfolding Mixed-Symmetry Fields in AdS and the BMV Conjecture: II. Oscillator Realization", JHEP 0907 (2009) 014, arXiv:0812.4438. 